\title{
DETERMINATION OF BRACHIARIA SPP. FORAGE QUALITY BY NEAR-INFRARED SPECTROSCOPY AND PARTIAL LEAST SQUARES REGRESSION
}

\author{
MARIEL MONRROY1,2, DEHYLIS GUTIÉRREZ ${ }^{1,2}$, MARISSA MIRANDA ${ }^{1,2}$, KARLA HERNÁNDEZ $^{3}$, \\ AND JOSÉ RENÁN GARCÍA ${ }^{1,2, *}$ \\ ${ }^{1}$ Centro de investigación en Bioquímica y Química Aplicada, Universidad Autónoma de Chiriquí, Panamá \\ ${ }^{2}$ Departamento de Química, Facultad de Ciencias Naturales y Exactas, Universidad Autónoma de Chiriquí, Panamá \\ ${ }^{3}$ Laboratorio de Bromatología, Instituto de Investigación Agropecuaria de Panamá
}

\begin{abstract}
Characterizing the chemical properties of forage is critical for the production of improved pastures and livestock development. Conventional analysis methods are very time- and material-consuming, whereas near-infrared spectroscopy (NIRS) and chemometric analyses allow a fast simultaneous determination of various chemical or physical properties without the use of solvents or large sample amounts. The present research involved the development of models based on NIRS and partial least squares regression (PLS) to estimate the neutral detergent fiber (NDF), acid detergent fiber (ADF), cellulose, and crude protein (CP) contents in Brachiaria spp. forage samples. The models were constructed using spectral data in the range of 800 to $1850 \mathrm{~nm}$. Different preprocessing methods were applied, such as standard normal variate and first-/second-derivative transformations. The obtained calibration models were internally cross-validated, displaying validation errors similar to those obtained for conventional methods. The predictive abilities of the developed models were evaluated for external set samples. NDF, ADF, cellulose, and CP contents were estimated with relative errors of prediction (REPs) of 1.8, 2.6, 4.1, and 8.5\%, respectively. NIRS predictions are a useful and profitable tool for fast multi-sample chemical property analysis that is required for the assessment of forage quality. The obtained models are suitable for estimating the key chemical characteristics of forage quality. This research contributes a new approach to determining the quality of Brachiaria spp. forage and provides a new technological tool for the improvement of this crop.
\end{abstract}

Keywords: forage, NIRS, partial least squares, chemical properties

\section{INTRODUCTION}

Forage is the main source of nutrients for the maintenance and growth of livestock. ${ }^{1}$ The quality of forage is related to its ability to provide livestock with required nutrients, being of key importance for meat and dairy producers, with crude protein $(\mathrm{CP})$, neutral detergent fiber (NDF), acid detergent fiber (ADF), and total fat contents used as indicators of quality. ${ }^{2}$

Effective livestock breeding depends on the amounts of CP, NDF, ADF, and fat in forage, with the determination of forage quality being essential for livestock nutrition. ${ }^{2,3}$ Traditional forage quality analysis involves timeconsuming and expensive wet-chemical techniques, restricting the number of samples that can be processed in a short period of time. Thus, to improve forage characterization, faster and more cost-effective methods are required for the rapid estimation of quality-related parameters. ${ }^{4}$

Near-infrared spectroscopy (NIRS) combined with chemometric analysis is a useful and powerful technique used for the quality control of various raw materials and products in food, agricultural, pharmaceutical, and petrochemical industries, ${ }^{5-7}$ e.g., in the chemical composition determination of bamboo and wood, ${ }^{8,9}$ soil organic matter analysis, ${ }^{10}$ wheat grain carbohydrate analysis, ${ }^{11}$ chemical property determination of cellulosic pulps, ${ }^{12}$ and material evaluation for bioethanol production. ${ }^{13,14}$

In contrast to conventional analysis methods, NIRS has the advantages of being nondestructive, requiring a small sample size and a short analysis time, not using chemical reagents, being environmentally friendly (since no waste is generated), and allowing various parameters to be determined at-/on-line offering a low-cost measurement alternative, ${ }^{4,7,15}$

NIRS has a high potential for the determination of parameters of interest in the livestock industry. This industry requires a constant evaluation of forage parameters in order for the animals to obtain high-quality food for proper development. Therefore, an increasing demand exists for the evaluation of feed material quality. Stuth et al. ${ }^{4}$ describe the use of NIRS for determining the protein, fiber, tannin, and mineral content in forage. Additionally, Landau et al. ${ }^{16}$ have shown the capability of NIRS to predict the chemical constituents of ruminant feeds, e.g., CP and cell wall composition. Nevertheless, the analysis of tropical forage by NIRS has not been extensively reported. ${ }^{17}$

Forage species of the genus Brachiaria spp. are most widely used in tropical regions, mostly in Africa, South America, and Latin America. ${ }^{3,18,19}$ This genus exhibits excellent adaptability to acid soils and temperature changes. ${ }^{3}$ Despite its good nutritional characteristics and adaptability, the quality of this forage greatly depends on environmental factors and soil fertility, which necessitates a constant assessment of nutritional value.
Therefore, the purpose of this study was to use Fourier transform (FT)-NIR spectroscopy coupled with partial least squares regression (PLS) to generate calibration models and estimate NDF, ADF, cellulose, and CP contents in Brachiaria spp. forage samples.

\section{EXPERIMENTAL}

\section{Raw material}

The Bromatology Laboratory of the Agro-Livestock Research Institute of Panamá provided 153 samples of Brachiaria spp. forage, which were air-dried until reaching a $10 \%(\mathrm{w} / \mathrm{w})$ moisture content and were subsequently stored in plastic bags.

Chemical characterization

Samples were analyzed to determine dry matter (DM), CP, NDF, $\mathrm{ADF}$, cellulose, and acid detergent lignin (ADL) contents. DM content was determined after drying at $105^{\circ} \mathrm{C}$. The micro-Kjeldahl method (Association of Official Analytical Chemists AOAC, Method 976.05) was used to determine the nitrogen content, with $\mathrm{CP}$ content calculated by multiplying the nitrogen content by $6.25(\mathrm{CP}=\mathrm{N} \times 6.25) .{ }^{20} \mathrm{NDF}, \mathrm{ADF}$, and ADL contents were determined according to the methods of Van Soest et al. ${ }^{21}$ using an ANKOM ${ }^{20}$ fiber analyzer (ANKOM Technology Corporation, NY, USA). Cellulose content was calculated as the difference of ADF and ADL. All samples were analyzed in triplicates.

FT-NIRS analysis

Samples were initially dried at $50{ }^{\circ} \mathrm{C}$ for $24 \mathrm{~h}$ and conditioned to $50 \%$ relative air humidity at $25{ }^{\circ} \mathrm{C}$ in the spectrophotometer room. Spectra were recorded in diffuse reflectance mode at $2-\mathrm{nm}$ intervals from 570 to $1850 \mathrm{~nm}$ using 32 scans of the InfraXactTM Lab instrument (FOSS, MA, USA). Two spectra were recorded and averaged for each sample. The raw reflectance data were converted to absorbance, with baseline correction performed by Essential FTIR software (Essential FTIR, USA).

\section{Multivariate calibration}

Calibration models were developed on the basis of PLS algorithms, linking the spectral data to the values provided by the chemical analysis for each property. The spectral data were preprocessed using standard normal variate (SNV) and first- and/or second-derivative methods. Prior to PLS analysis, the final preprocessed spectral data were mean-centered. All models were internally and externally validated. The internal validation was based on full cross-validation (CV). A group of 153 samples was analyzed by FT-NIRS, with 123 samples used to construct the calibration models and 30 samples randomly 
selected from the whole group for external validation (prediction set).

Calibration accuracy was expressed by the root mean square error of calibration (RMSEC, determined from the residuals of the final calibration), the root mean square error of cross-validation (RMSECV, determined from the residuals of each cross-validation phase), and the coefficient of determination $\left(r_{\mathrm{c}}^{2}\right.$, defined as the proportion of variation in the calibration set explained by the model).

For external validation, the standard error of prediction (SEP, determined from the residuals of the predictions), bias (average difference between $\hat{y} i$ and $y i$, where $\hat{y} i$ is the property content for a sample $i$ predicted by the calibration model; $y i$ is the known property content of an external sample $i$ ), root mean square error of prediction (RMSEP), and relative error of prediction (REP, determined by the percentage ratio between RMSEP and average experimental value) were calculated. The predictive abilities of developed calibration models were also assessed by calculating the coefficient of determination $\left(r^{2}\right.$, defined as the proportion of variation in the independent prediction set that was explained by the calibration). Multivariate analyses were performed using Software Pirouette v. 4.0 (Infometrix, USA)

\section{RESULTS AND DISCUSSION}

The main forage quality parameters were evaluated in 153 samples of Brachiaria spp, with the results summarized in Table 1. NDF, FDA, cellulose, and $\mathrm{CP}$ contents were in the ranges of $62.7-85.7 \%, 28.5-45.5 \%, 25.6-42.3 \%$, and $5.6-11.1 \%$, respectively. The chemical composition of Brachiaria spp forages was thus similar to that reported for silage and forage from alfalfa hay and maize. ${ }^{22-24}$

Table 1. Summary of parameters evaluated in Brachiaria spp. forages by conventional methods.

\begin{tabular}{|c|c|c|c|c|c|c|}
\hline Parameter & Mean & Minimum & Maximum & SD & Minimal SD & Maximal SD \\
\hline NDF (\%) & 76.6 & 62.7 & 85.7 & 1.1 & 0.1 & 3.3 \\
\hline ADF (\%) & 38.9 & 28.5 & 45.5 & 0.8 & 0.1 & 3 \\
\hline Cellulose (\%) & 35.7 & 25.6 & 42.3 & 0.6 & 0.01 & 2.3 \\
\hline CP (\%) & 8.4 & 5.6 & 11.1 & 0.3 & 0.004 & 1 \\
\hline
\end{tabular}

Note: Percentages are presented on DM basis.

The evaluated chemical properties are indicators of forage quality, with their variation range being wide enough for the development of NIRS models. For this reason, prediction models based on FT-NIRS and chemometric analysis were developed for CP, NDF, ADF, and cellulose. The spectral data were transformed to their first and second derivatives (as described in the experimental section) to eliminate variations such as baseline shifts and noise and to highlight modifications that affect the effective calibration of relationships between structural characteristics and NIR spectra (Figure 1).
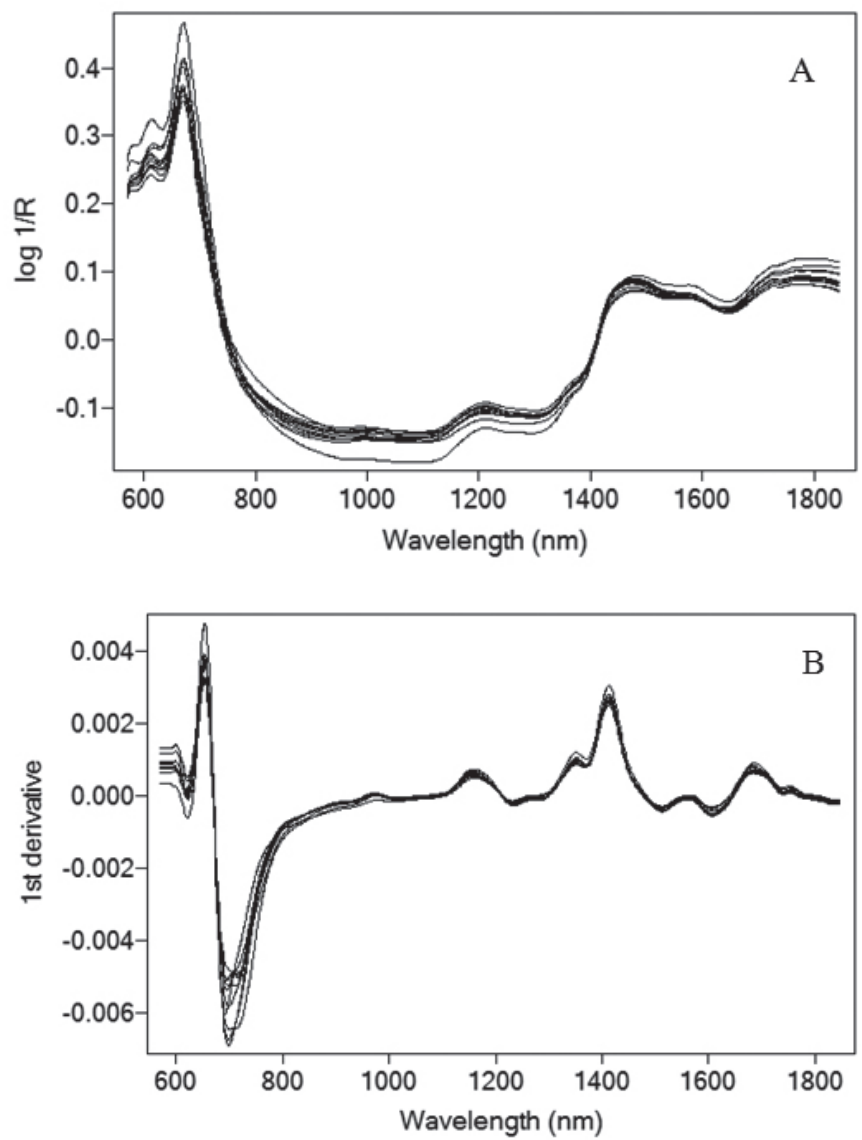

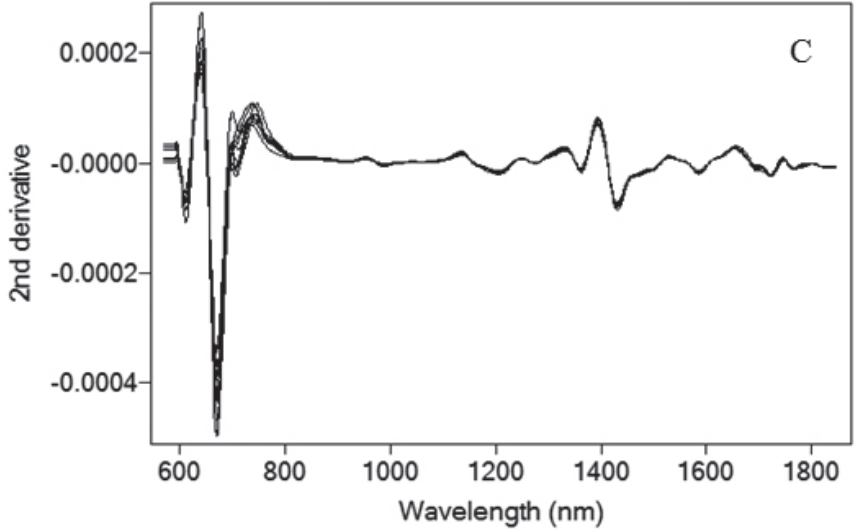

Figure 1. FT-NIR spectra of pretreated materials. Raw reflectance (R) data were converted to absorbance units $(1 / R)(A)$ and transformed using the first (B) and second derivative (C).

The calibration model was operated using principal component regression (PCR) and PLS algorithms and SNV, smooth, first- and second-derivative transformations, generating a large number of models for each property evaluated. The best models (having a small number of factors, small RMSECV, high $r_{\mathrm{c}}^{2}$, and better predictive ability) were obtained using the PLS algorithm. Some outliers were detected based on leverage and studentized residuals and were removed. Table 2 summarizes the statistical parameters of calibration and cross-validation for the best models. The variance $\left(r_{c}^{2}\right)$ exhibited by these models varied between 0.65 and 0.83 . The RMSECV values were comparable to those of RMSEC for all calibrations, indicating that the excluded sample was well predicted by the corresponding calibration in each cross-validation step and that the RMSEC values were not overly optimistic, denoting the roughness of the models. The models obtained for NDF and cellulose required preprocessing of the spectral data using SNV and first-derivative transformations, affording RMSECV values of 1.9 and $1.3 \%$ with five factors, respectively. For ADF and CP, using PLS models resulted in RMSECVs of 1.5 and $0.8 \%$ with four and seven factors, respectively. The corresponding spectral data preprocessing used SNV and second-derivative transformations in both cases.

Other studies have also demonstrated the potential of NIRS for predicting forage and silage quality. Campo et al..$^{22}$ evaluated modified partial least squares (MPLS) and PLS models for the estimation of NDF, ADF, and CP contents in Zea mays forage and reported RMSECVs of 1.7, 1.0, and 0.3\%, respectively. Lundberg et al. ${ }^{24}$ predicted these properties for legume grass and 
Zea mays silage, obtaining RMSECVs of 1.7 and $1.8 \%$ for NDF, 2.2 and $1.4 \%$ for ADF, and 0.3 and $0.8 \%$ for CP, respectively. Additionally, Castro et al. ${ }^{25}$ developed MPLS models for green herbage forage and silage, obtaining RMSECVs of 2.6 and $2.8 \%$ for NDF, 1.2 and $2.1 \%$ for ADF, and 0.66 and $1.3 \%$ for CP, respectively. To the best of our knowledge, no models for estimating cellulose content in silages and forages using NIRS have been developed. Nevertheless, estimation of this property by NIRS has been reported for wood samples. For example, Hodge and Woodbridge ${ }^{26}$ developed NIRS models for the cellulose content of different pine species with an RMSECV of $1.1 \%$.

Table 2. Summary of calibrations for the developed FT-NIRS models with corresponding statistical parameter values for the best models.

\begin{tabular}{|c|c|c|c|c|}
\hline Property & NDF (\%) & ADF (\%) & Cellulose (\%) & CP (\%) \\
\hline Transformation of data & $1^{\text {st }}$ der. (15), SNV & $2^{\text {nd }}$ der. $(23)$, SNV & $1^{\text {st }}$ der. (21), SNV & $2^{\text {nd }}$ der. (23), SNV \\
\hline Range & $63-86$ & $28-45$ & $25-42$ & $5.6-11.3$ \\
\hline Spectral region (nm) & $800-1850$ & $800-1850$ & $800-1850$ & $\begin{array}{c}628-792,110-1220,1314-1734, \\
1846-1848\end{array}$ \\
\hline Number of PLS factors & 5 & 4 & 5 & 7 \\
\hline Number of outliers & 1 & 2 & 1 & 121 \\
\hline $\boldsymbol{n}^{2}$ & 122 & 121 & 122 & 0.65 \\
\hline $\boldsymbol{r}_{\mathbf{c}}$ & 0.87 & 0.88 & 0.90 & 0.71 \\
\hline RMSEC $_{\boldsymbol{r}_{\mathrm{v}}{ }^{2}}$ & 1.71 & 1.36 & 1.26 & 0.56 \\
\hline RMSECV & 0.84 & 0.86 & 0.88 & 0.78 \\
\hline
\end{tabular}

Note: The values in parentheses correspond to the number of points used in the data transformation.

Table 3. Summary of predictions (external validation).

\begin{tabular}{|c|c|c|c|c|c|c|c|c|}
\hline \multirow{2}{*}{ Property } & \multirow{2}{*}{ Range } & \multicolumn{9}{|c|}{ External validation } \\
\cline { 3 - 10 } & & $\boldsymbol{n}$ & $\boldsymbol{r}_{\mathbf{p}}{ }^{2}$ & RMSEP & SEP & \multicolumn{2}{|c|}{ REP } & \multicolumn{2}{c|}{ BIAS } & \multicolumn{2}{|c|}{ RPD } \\
\hline NDF & $68-83$ & 30 & 0.86 & 1.4 & 1.4 & 1.8 & 0.05 & 3.4 \\
\hline ADF & $30-44$ & 29 & 0.90 & 1.0 & 1.0 & 2.6 & 0.07 & 5 \\
\hline Cellulose & $29-42$ & 30 & 0.73 & 1.4 & 1.4 & 4.1 & 0.42 & 2.6 \\
\hline CP & $6-10$ & 30 & 0.53 & 0.71 & 0.72 & 8.5 & 0.1 & 3.5 \\
\hline
\end{tabular}

The use of NIRS to estimate tropical forage quality has been less studied. Baloyi et al. ${ }^{17}$ reported one of the few studies on the estimation of the chemical composition of tropical forage using this technique. The authors performed NIRS modeling for NDF, ADF, and CP, obtaining RMSECV values of 1.7 1.7 , and $1.2 \mathrm{~g} / \mathrm{kg}$, respectively, for Vigna unguiculata, Desmodium uncinatum, Stylosanthes guianensis, and other forage.

In general, calibration models similar to or better than those reported were developed in the present study to predict $\mathrm{CP}, \mathrm{NDF}, \mathrm{ADF}$, and cellulose contents in samples of Brachiaria spp. forage from Panamá.

The calibration models were used to predict the properties of 30 independent samples in an external validation set. External validation models provided an estimate of how well a regression model would perform if it were applied to unknown samples. The validation set covered the whole range of property values, with the results of prediction assays summarized in Table 3 and Figure 2. The NDF, FDA, cellulose, and CP contents were predicted with RMSEPs of $1.4,1.0,1.4$, and $0.7 \%$, respectively. The RMSEP values were similar or slightly less than the corresponding RMSECVs, ratifying the good predictive ability of these models in internal validation. Although the RMSEP were greater than the average standard deviation obtained for wet-chemical analysis, they were still in the range of standard deviations commonly obtained in the analysis of these forage chemical properties. This indicated that the above models are appropriate to estimate these parameters, which are of key importance to evaluate forage quality. Moreover, NDF, FDA, cellulose, and CP contents were predicted with REPs of $1.8,2.6,4.1$, and $8.5 \%$, respectively. According to Olivieri and Escandar, ${ }^{27}$ REP values of less than $5 \%$ are considered good, with those between 5 and $10 \%$ considered reasonable. This shows the quality of the developed prediction models. Even though, a moderate accuracy for $\mathrm{CP}$ content predictions can be expected, the results indicate the existence of a difference between the experimental and estimated values for $\mathrm{CP}$ content $\left(r^{2}=0.53\right)$, possibly due to the narrow range of sample concentrations used in the calibration procedure.

Additionally, the bias and relative performance determinant (RPD) parameters were evaluated. As shown in Figure 2, the calculated bias was low, indicating accurate prediction and explaining the similar SEP and RMSEP values. According to Saeys et al., ${ }^{28}$ the predictive ability for RPD can be considered successful, since values higher than 2.5 were obtained.

On the other hand, the regression vectors of PLS models were analyzed to determine the functional groups responsible for the relationships between each property and sample spectra. These vectors are shown in Figure 3 Assigning molecular features to NIR spectra is difficult due to band overlap, but some tentative assignments can still be made. ${ }^{7,29-32}$ In the NDF model, the most important bands were observed at 1168 and $1216 \mathrm{~nm}$ (tentatively indicative of the second $\mathrm{C}-\mathrm{H}$ overtone), $1436 \mathrm{~nm}$ (first $\mathrm{O}-\mathrm{H}$ overtone and/or $\mathrm{C}-\mathrm{H}$ combination), $1472 \mathrm{~nm}$ (first $\mathrm{O}-\mathrm{H}$ overtone), and 1608, 1680, and 1768 $\mathrm{nm}$ (C-H stretch first overtone). These bands were also most important for the development of the cellulose model. In the ADF model, the corresponding bands were observed at $1450 \mathrm{~nm}$ (first $\mathrm{O}-\mathrm{H}$ overtone), 1504 and $1590 \mathrm{~nm}$ (O-H stretch first overtone), and $1696 \mathrm{~nm}$ (C-H stretch first overtone). For the CP model, the influential bands were observed at $728 \mathrm{~nm}$ (associated with red color in the visible region); $1188 \mathrm{~nm}$ (second $\mathrm{C}-\mathrm{H}$ overtone), $1366 \mathrm{~nm}$ (C-H combination), $1478 \mathrm{~nm}(\mathrm{~N}-\mathrm{H}$ stretch first overtone), and 1666 and 1696 $\mathrm{nm}(\mathrm{C}-\mathrm{H}$ stretch first overtone). These assignments are consistent with the chemical characteristics of lignocellulosic materials.

According to the obtained results, NIRS-PLS models can satisfactorily estimate the NDF, ADF, and cellulose contents in Brachiaria spp. forage. The numerous advantages offered by NIRS make it a promising analysis technique. Improvements in instrumentation and the advance of chemometric software allow simultaneous quick and easy analyses of a variety of parameters. Moreover, sample preparation is not as essential for NIRS as it is for conventional analyses techniques. Nonetheless, an extensive knowledge of chemometrics is required. 

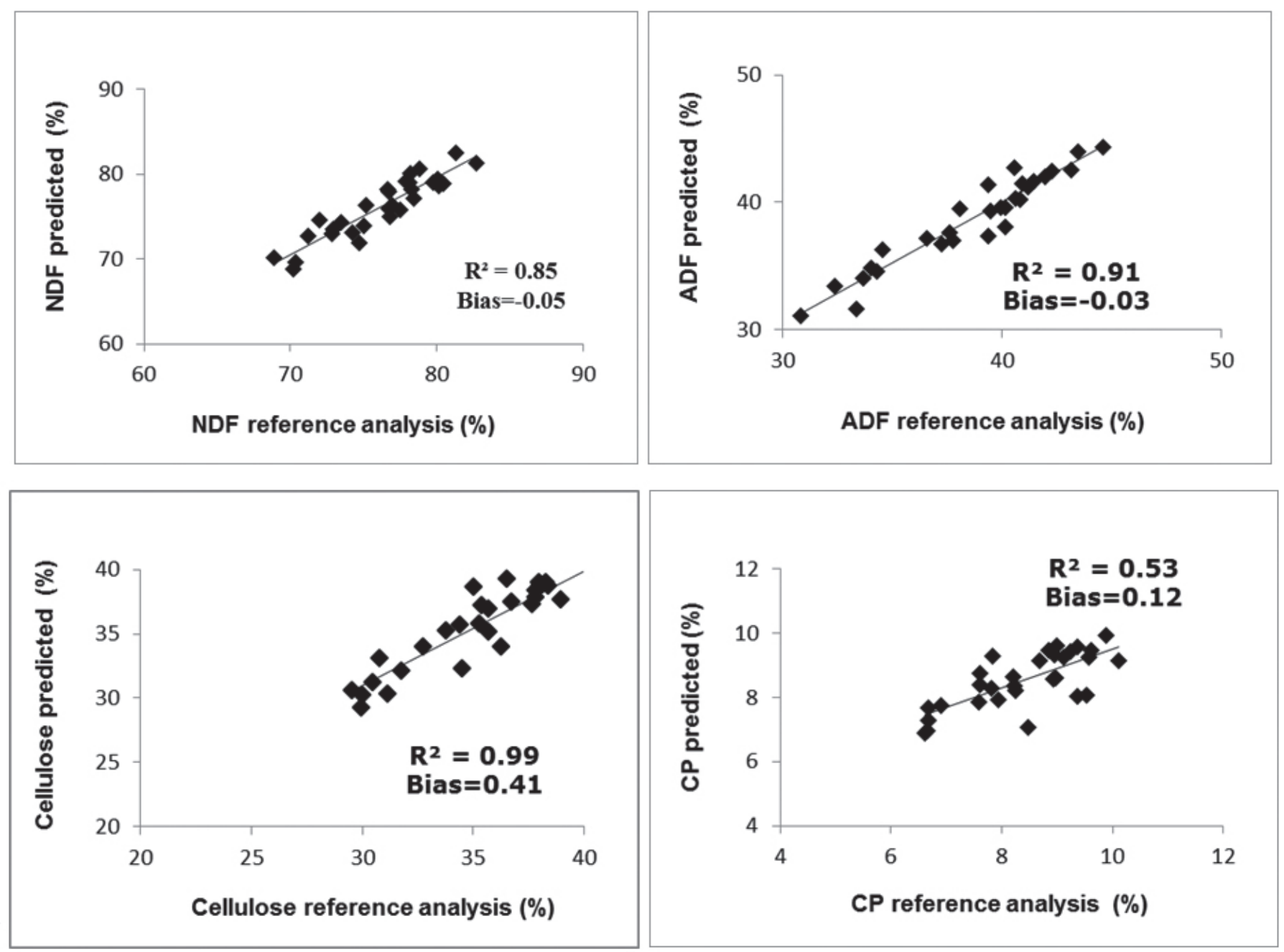

Figure 2. Correlations between observed and PLS-predicted concentrations for 30 samples evaluated as a validation data set.

\section{CONCLUSION}

The obtained calibration models showed validation errors similar to those obtained for conventional methods, proving the suitability of NIRS for predicting the chemical properties of Brachiaria spp. forage. This technique can be an attractive alternative for replacing the time-consuming reference methods and decreasing the cost of chemicals used, being an environmentally friendly and profitable tool for the rapid analysis of a large number of samples required in the assessment of the nutritional quality of tropical forages, such as Brachiaria spp.

\section{ACKNOWLEDGEMENTS}

Financial support was received from SENACYT as a member of the Panamá Research National System (SNI). Additionally, the authors acknowledge the collaboration of the Bromatology Laboratory of the AgroLivestock Research Institute of Panama. 

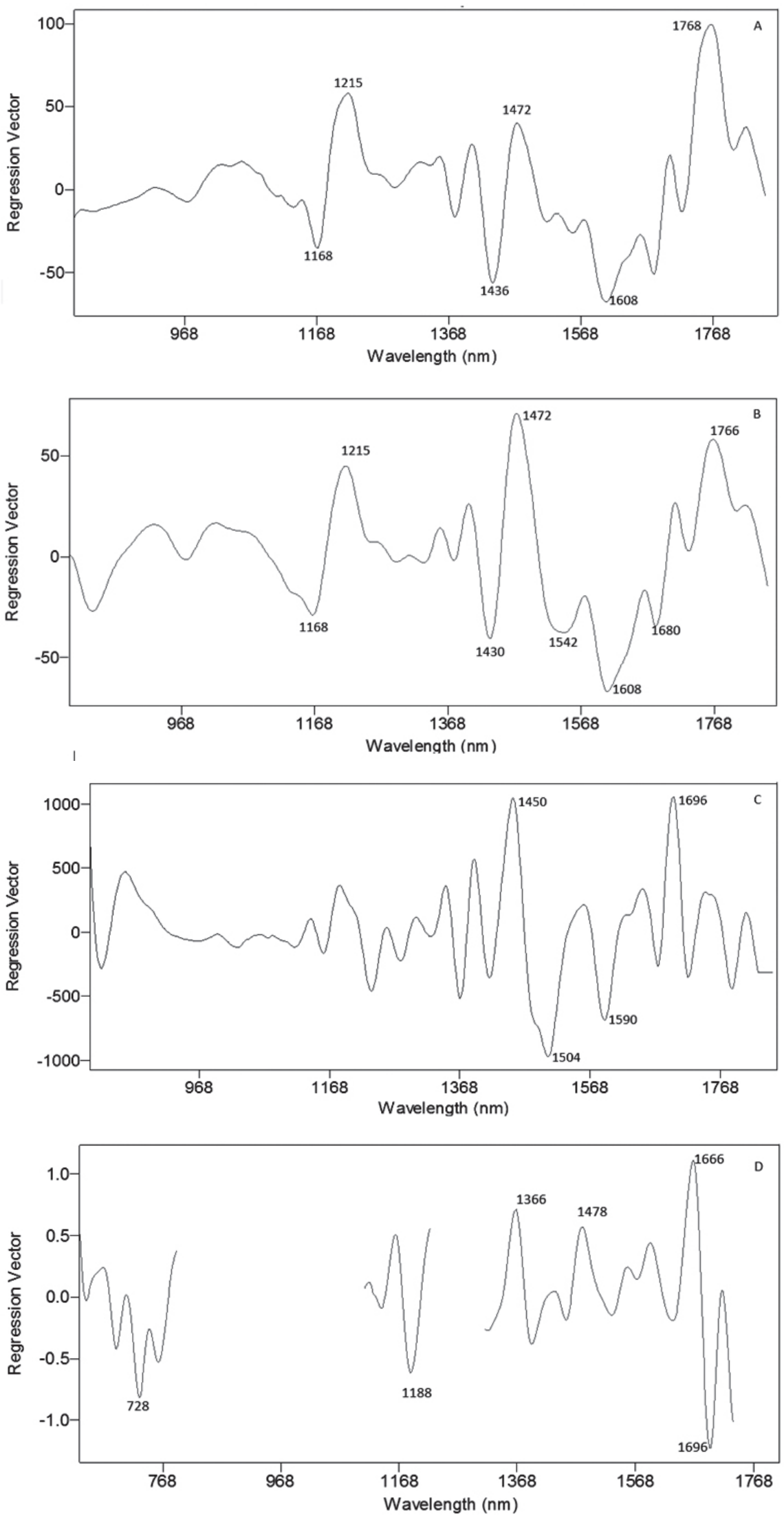

Figure 3. Regression vectors of PLS models used to estimate NDF (A), cellulose (B), ADF (C), and CP (D) content in Brachiaria spp. forages. 


\section{REFERENCES}

1. H. Zaker, H. Aminpanah, H. Darkhal, Agriculture and Forestry 60, 193, (2014)

2. F. Amiri, A. Shariff, M. Rashid, Songklanakarin J. Sci. Technol. 34, 577 (2012).

3. A. F. Ribeiro, J. D. Messana, P. H. M. Dian, R. A. Reis, A. C. Ruggieri, E. B. Malheiros, T. T. Berchielli, Ital. J. Anim. Sci. 13, 36, (2014).

4. J. Stuth, A. Jama, D. Tolleson, Field Crop Res. 84, 45, (2003).

5. V. Bellon, J. L. Vigneau, F. Sévila, Food Control 5, 21, (1994).

6. H. Huang, H. Yu, H. Xu, Y. Ying, J. Food Eng. 87, 303, (2008).

7. H. W. Siesler, Y. Ozaki, S. Kawata, H. M. Heise Near-Infrared Spectroscopy: Principles, Instruments, Applications, $1^{\text {st }}$ ed. Wiley-VCH, Weinheim, 2002.

8. Y. Sun, L. Lin, H. Deng, J. Li, B. He, R. Sun, P. Ouyang, BioResources 3 , 297, (2008)

9. Z.-H. Jiang, Z. Yang, C.-L. So, C.-Y. Hse, J. Wood Sci. 53, 449, (2007).

10. M. St. Luce, N. Ziadi, B. J. Zebarth, C. A. Grant, G. F. Tremblay, E. G. Gregorich, Geoderma 232, 449, (2014).

11. A. Salgó, S. Gergely, J. Cereal Sci. 56, 31, (2012).

12. M. Monrroy, R. T. Mendonça, J. Ruiz, J. Baeza, J. Freer, J. Wood Chem. Technol. 29, 150, (2009).

13. R. Castillo, J. Baeza, J. Rubilar, Á. Rivera, J. Freer, Appl. Biochem. Biotechnol. 168, 2028, (2012).

14. M. Monrroy, J. R. Garcia, E. Troncoso, J. Freer, J. Chem. Technol. Biot. 90, 1281, (2015)

15. Y.-H. Jia, X.-P. Liu, Y.-C. Feng, C.-Q. Hu, AAPS PharmSciTech 12, 738, (2011).

16. S. Landau, T. Glasser, L. Dvash, Small Ruminant Res. 61, 1, (2006).

17. J. J. Baloyi, H. Hamudikuwanda, N. Berardo, M. Ordoardi, N. T. Ngongoni, Afr. J. Agric. Res. 8, 868, (2013).

18. A. M. De Souza-Kaneshima, C. Simioni, M. F. Felismino, A. B. Mendes-
Bonato, C. Risso-Pascotto, C. Pessim, M. S. Pagliarini, C. B. Do Valle, Plant Breeding 129, 186, (2010).

19. E. R. Canchila, M. Soca, F. Ojeda, R. Machado, N. Canchila, Pastos y Forrajes 33, 7, (2010).

20. AOAC, in Official Methods of Analysis $\left(18^{\text {th }}\right)$, AOAC International, Arlington, VA 2005

21. P. J. Van Soest, J. B. Robertson, B. A. Lewis, J. Dairy Sci. 74, 3583, (1991).

22. L. Campo, A. B. Monteagudo, B. Salleres, P. Castro, J. Moreno-Gonzalez, Span. J. Agric. Res. 11, 463 (2013).

23. R. Kowsar, G. R. Ghorbani, M. Alikhani, M. Khorvash, A. Nikkhah, J. Dairy Sci. 91, 4755, (2008)

24. K. M. Lundberg, P. C. Hoffman, L. M. Bauman, P. Berzaghi, The Professional Animal Scientist 20, 262, (2004).

25. P. Castro in Lowland and Grasslands of Europe: Utilization and Development, G. Fisher and B. Frankow-Lindberg eds. Food and Agriculture Organization of the United Nations (FAO), Rome, 2002; pp. 225-22826. G. R. Hodge, W. C. Woodbridge, J. Near Infrared Spec. 18, 367, (2010).

27. A. C. Olivieri and G. M. Escandar in Practical Three-Way Calibration, Elsevier, Boston, 2014; pp. 65-92.

28. W. Saeys, A. M. Mouazen, H. Ramon, Biosyst. Eng. 91, 393, (2005).

29. P. Jones, L. R. Schimleck, G. Peter, R. Daniels, A. Clark, Wood Sci. Technol. 40, 709, (2006)

30. S. S. Kelley, T. G. Rials, R. Snell, L. H. Groom, A. Sluiter, Wood Sci. Technol. 38, 257, (2004).

31. J. Workman, L. Weyer, Practical Guide to Interpretive Near-Infrared Spectroscopy, Taylor \& Francis Group, New York and Florida, 2008.

32. Shenk, J. Workman, M. Westerhaus in Handbook of Near-Infrared Analysis, D. Burns, E. Ciurczak eds. CRC Press, New York and Florida, 2008; pp. 34-32. 